\title{
Does Rural to Urban Migration Improves Indian Women's Health Status and Knowledge of AIDS
}

\author{
Sutapa Agrawal $^{1^{*}}$,Praween Agrawal $^{2}$ \\ ${ }^{1}$ South Asia Network for Chronic Disease, Public Health Foundation of India, New Delhi, India \\ ${ }^{2}$ Population Council, New Delhi, India \\ Abstract
}

The present paper explores the effect of patterns and duration of migration upon health and morbidity condition of women and knowledge and awareness of AIDS. Cross-sectional data from India's second National Family Health Survey (NFHS-2, 1998-99) is used for this study. Analysis is based on 73,558 women age 15-49 years who belonged to different streams of migration. Bivariate as well as multivariate techniques have been used for data analysis. Women migrating towards rural area are more underweight than migrating towards urban area whereas reverse for overweight. However, women migrating from rural to rural area were more anaemic than women migrating from rural to urban area. Significant differences were also found for morbidity conditions like Asthma, Tuberculosis, Jaundice, Malaria and some reproductive health problems according to streams of migration. Knowledge of AIDS also significantly differs according to the stream and duration of migration. We found stream of migration and duration of migration plays a key role in health, morbidity condition and knowledge of AIDS among women. Therefore, quality of health care in urban areas should be more widely disseminated in rural areas to improve the health status of women. Also the information-education-communication (IEC) programmes related to AIDS should be made more strengthened and effective through television, radio and also through the school teachers to reach the rural masses in India.

Key Words: migration pattern; duration; health; morbidity; underweight; overweight; anaemia; reproductive health; AIDS; women' India

\section{INTRODUCTION}

$\mathrm{I}$ $\mathrm{n}$ the contemporary developing world, population mobility in the form of rural to urban migration, has been increasing rapidly since the 1960 s. During the past three decades migration in the developing world has not only increased in scale, but also in the diversity of the groups involved, and the moves themselves are becoming increasingly complex in the nature of their spatial patterning.[1] India has witnessed a threefold increase in population mobility levels, from $11 \%$ of the total population classified as migrants in 1950 to $31 \%$ in 1981.[2] In $1981,71 \%$ of all migrants had rural destinations (65\% ruralrural and $6 \%$ urban-rural). This figure is a slight decline from 1971, where $75 \%$ of all migrants were rural to rural migrants.[3] At the intra-state level, females dominate the rural to rural stream, accounting for more than two-thirds of all migrants due to the prevalence of patrilocal marriages.[4] The trend remains similar even in 2001 census. [5]

Migration in India has traditionally been dominated by short-term rural to rural movements, which accounts for more than $60 \%$ of all migration, and are comprised mainly of women moving between their natal and marital homes upon marriage.[6] The process of early marriage and the tradition of marriages between people from nearby villages and territorial endogamy has resulted in both early adult responsibilities and reduced the need to migrate long distances.[7] Weiner[8] showed that the Indian marriage market has become much wider, with longer distances apparent in the marriage system. A product of this has been an increase in the distances involved in rural to rural migration, with an increase in the number of inter-state ruralbound migrations. The remaining combinations of rural and

\section{Address for correspondence* \\ Sutapa Agrawal $\mathrm{PhD}$, \\ South Asia Network for Chronic Disease (SANCD), Public \\ Health Foundation of India (PHFI), First Floor, C-1/52 \\ Safdarjung Development Area, New Delhi 110016, INDIA. E- mail: sutapaiips@ rediffmail.com or sutapa.agrawal@phfi.org}

urban migration (urban to rural and urban to urban) continue to form only minor aspects of the migration system in India. Urban to urban migration has continued to account for approximately 5\% of the total migration since the increase in urbanisation during the 1960 's.6 A majority of urban to urban migration is in the form of an upward movement through the urban hierarchy (as suggested in Ravenstein's Laws of Migration, 1885).[9]

Brockerhoff[10-12] has used the results of Demographic and Health Surveys to analyse the relationship between mortality and rural to urban migration in Sub-Saharan Africa. The results confirm those of earlier epidemiological studies, which suggested that, migrants had higher mortality than urban natives and lower mortality than rural sedantes. Brockerhoff and Eu[13] demonstrated that rural to urban migration is selective of the younger, higher educated members of the rural population. Female migrants tend to be younger than their male counterparts due to the practice of patrilocal marriage migration.[14] It has been shown that the propensity to migrate to an urban area is highest among educated people in rural areas.[15] The rural areas may hold greater economic security for the unskilled and uneducated for whom employment is scarce in the cities.[16] Successful adaptation to the urban environment depends not only on the behaviour and social mobility of the migrants, but also on the receptivity of the urban society.[11]

Although our knowledge of the relationship between migration and health of women is increasing, we still have a limited knowledge of migration and health of women in the reproductive ages.[17 ]Also, there is a dearth of study, which tells about the health condition and knowledge of AIDS of migrant women according to the pattern of migration in India. In the present study large-scale survey data gives a scope to study about the different health aspects of migrant women of reproductive age group according to the pattern of migration along with their socioeconomic and demographic characteristics. The specific objectives of the study are: to study the socio-economic and demographic characteristics of migrant women by patterns of 
migration; to investigate the effect of patterns of migration upon health and morbidity condition of women, with a special focus on maternal and reproductive health and to examine the knowledge and awareness of AIDS among migrant women according to their pattern of migration and duration of stay.

\section{METHODS}

DataisfromIndia'ssecondNationalFamilyHealthSurvey (NFHS-2) conducted during 1998-99. This survey was designed on the lines of the Demographic and Health Surveys (DHS) that have been conducted in many developing countries since the 1980s. NFHS-2 collected demographic, socio-economic, and health information from a nationally representative probability sample of 90,303 ever-married women aged 15-49 years residing in India's 92,486 households. All the states of India are represented in the sample (except the small Union Territories), covering more than $99 \%$ of country's population. The sample is a multi-stage random sample with an overall response rate of $98 \%$. Details of sample design, including sampling framework and sample implementation, are provided in the basic survey report for all India.[18].

We have used the information on the type of place of residenceandtypeofplaceofpreviousresidenceforidentificationof migrant women. From the information about the rural-urban residence, thefourstreamsofmigration(ruraltorural,ruraltourban, urban to rural and urban to urban) have been categorised. While categorisation of the stream of migration, visitors have been excluded from the sample. A total of 73,558 women from different streamsofmigrationisincludedfortheanalysis.Alsonon-migrating women had been separately analysed as a control group for comparison purpose. Atotal sample of 10,161 non-migrant women was found consisting of 6,687 rural non-migrants and 3,474 urban non-migrants. NFHS-2 provides detailed information on nutrition, fertility, family planning, morbidity, maternal and reproductive healthofwomenandknowledgeofAIDS, whichareimportantinputs forthestudy.

\section{Statisticalanalysis}

Bivariate as well as multivariate techniques have been usedfordata analysis. Inbivariateanalysis, crosstabulationisdone ineachofthefourcategorieswithdifferentdependentvariablessuch as socio-economic and demographic characteristics of individual. Inmultivariateanalysis, binarylogisticregressionanalysishasbeen restored to know the odds ratio of each socio-economic and demographic characteristic of women, controlling for others, according to different streams of migration.

\section{RESULTS}

Socio-economic and demographic characteristics of women by patterns of migration:All the four patterns of migration had been found in case of Indian women. Overall, rural to rural migration (69\%) ranks first followed by urban to urban (13\%) and rural to urban migration (12\%) (data not shown). However, urban to rural migration accounts for only $6 \%$ of total migration. Table 1 presents selected socio-economic, demographic characteristics of women aged 15-49 years according to streams of migration and also among non-migrants. More percentage of younger women (aged 15-24 years) were found among rural to rural and urban to rural migrants than urban to urban and rural to urban migrants. More than $27 \%$ women migrates towards rural area in younger age (15-24 years) whereas one-fifth from rural to urban area and 18\% from urban to urban area. On the other hand, more proportion of relatively older women (aged 35-49 years) migrated towards urban area than rural area. However, more proportion of rural non-migrants (27\%) was from younger age group (15-24 years) than urban non-migrants $(20 \%)$.

Educational status of women is significantly associated with pattern of migration. More than $70 \%$ of rural to rural migrants were illiterate compared to only one in four among urban to urban migrants. The educational level of women migrating towards urban area was higher compared to women migrating towards rural areas, as $44 \%$ women who were migrating from urban to urban area were having education of high school and above compared to only $6 \%$ women who migrates from rural to rural area. Husband's educational status also shows similar situation with migration pattern but with little less gap. About $38 \%$ of rural to rural migrant women's husband were illiterate compared to $11 \%$ of urban to urban migrant women's husband. Also, only one-fifth of rural to rural migrant women's husband had a education of high school and above compared to more than half of urban to urban migrant women's husband. Moreover, educational status were found almost similar for rural to urban and urban to rural stream of migration of women. On the other hand, educational status of rural non-migrant women was found worse than women in any streams of migration. However, educational status of urban non-migrant women was in between the urban to urban and urban to rural migrants.

Religious differential was also found with the pattern of migration of women. More proportion of Hindu women were migrating from rural to rural area than other streams of migration whereas Muslim women were migrating more from urban to urban area than other streams of migration. $85 \%$ percent of rural to rural migration takes place among Hindu women compared to $80 \%$ from rural to urban area and three-fourth from urban to urban area. On the other hand, $17 \%$ Muslim women experienced urban to urban migration whereas $13 \%$ from rural to urban area and one in ten in case of rural to rural migration. However, women belonging to religions like, Sikh, Christianity and others were more prone to migrate towards urban area than rural area. In the distribution of non-migrant women, Sikh women were significantly less than that of migrant's category, whereas Christian women were significantly more among the nonmigrants than any migration stream category. However, distribution of Hindu and Muslim urban non-migrant women were quite disproportionate to migrant category. Significantly, a higher proportion of Muslim women were found among urban non-migrants than other migration streams whereas relatively lower proportion of Hindu women were found among the urban non-migrants than any other migration steams.

Ethnic differential also exists in the pattern of migration of women. More proportion of scheduled caste and scheduled tribes women migrate from rural to rural areas than urban to urban areas whereas women belonging to other castes migrates more from urban to urban areas than other streams. The proportion of scheduled caste women, migrating from rural to rural area, decreases from $20 \%$ to $12 \%$ in case of urban to urban migration. Similarly, the proportion for scheduled tribe women migrating from rural to rural area was $11 \%$, which decreases to $5 \%$ in case of urban to rural and further only to $2 \%$ in case of migration from urban to urban area. However, the proportion of other caste women in migration increased from $35 \%$ in rural to rural area migration, to $62 \%$ percent in case of urban to urban migration. On the other hand, more proportion of other backward class women were found among urban as well as rural non-migrants and less 
Table 1: Percent distribution of migrant women aged 15- 49 years according to streams of migration and non-migrant women by selected socio-economic and demographic characteristics, India, 1998-99

\begin{tabular}{|c|c|c|c|c|c|c|}
\hline \multirow[b]{2}{*}{ Background characteristics } & \multicolumn{4}{|c|}{$\begin{array}{c}\text { Migrants according to stream of } \\
\text { migration }\end{array}$} & \multicolumn{2}{|c|}{ Non-migrant } \\
\hline & $\begin{array}{c}\text { Rural } \\
\text { to } \\
\text { Rural }\end{array}$ & $\begin{array}{c}\text { Rural } \\
\text { to } \\
\text { Urban }\end{array}$ & $\begin{array}{c}\text { Urban } \\
\text { to } \\
\text { Rural } \\
\end{array}$ & $\begin{array}{c}\text { Urban } \\
\text { to } \\
\text { Urban } \\
\end{array}$ & $\begin{array}{c}\text { Urban } \\
\text { non- } \\
\text { migrant }\end{array}$ & $\begin{array}{c}\text { Rural non- } \\
\text { migrant }\end{array}$ \\
\hline \multicolumn{7}{|l|}{ Age groups } \\
\hline $15-24$ & 27.0 & 20.8 & 28.9 & 18.3 & 19.7 & 26.7 \\
\hline $25-34$ & 37.1 & 37.8 & 39.3 & 37.9 & 38.9 & 34.7 \\
\hline $35-49$ & 35.8 & 41.4 & 31.8 & 43.8 & 41.3 & 38.6 \\
\hline \multicolumn{7}{|l|}{ Education $^{1}$} \\
\hline Illiterate & 70.4 & 46.4 & 39.5 & 23.0 & 33.5 & 68.7 \\
\hline Literate, $<$ middle school complete & 17.6 & 23.7 & 25.3 & 19.6 & 24.2 & 18.9 \\
\hline Middle school complete & 6.0 & 10.8 & 13.4 & 13.0 & 12.0 & 6.0 \\
\hline High school complete and above & 5.9 & 19.1 & 21.8 & 44.4 & 30.3 & 6.4 \\
\hline \multicolumn{7}{|l|}{ Husband's education } \\
\hline Illiterate & 38.0 & 19.3 & 19.7 & 10.5 & 18.8 & 45.4 \\
\hline Literate, $<$ middle school complete & 27.7 & 25.1 & 25.7 & 17.7 & 23.9 & 26.8 \\
\hline Middle school complete & 13.4 & 14.8 & 14.6 & 13.0 & 12.9 & 10.2 \\
\hline High school complete and above & 20.9 & 40.8 & 39.9 & 58.8 & 44.3 & 17.7 \\
\hline \multicolumn{7}{|l|}{ Religion } \\
\hline Hindu & 84.8 & 80.7 & 81.3 & 74.5 & 69.5 & 80.9 \\
\hline Muslims & 10.8 & 12.6 & 12.1 & 17.3 & 23.2 & 12.6 \\
\hline Christian & 1.8 & 3.1 & 3.1 & 3.0 & 5.2 & 5.5 \\
\hline Sikh & 1.9 & 1.3 & 1.8 & 2.2 & 0.5 & 0.1 \\
\hline Others $^{2}$ & 0.7 & 2.3 & 1.8 & 2.9 & 1.7 & 1.0 \\
\hline \multicolumn{7}{|l|}{ Ethnicity } \\
\hline Scheduled caste ${ }^{3}$ & 20.4 & 17.9 & 17.5 & 11.9 & 15.1 & 18.1 \\
\hline Scheduled tribe ${ }^{3}$ & 11.1 & 4.4 & 5.4 & 2.5 & 4.7 & 13.3 \\
\hline Other backward class & 33.3 & 33.5 & 34.0 & 23.9 & 40.2 & 41.2 \\
\hline Others & 35.2 & 44.2 & 43.1 & 61.6 & 39.9 & 27.4 \\
\hline \multicolumn{7}{|l|}{ Occupation } \\
\hline Professional/Technical/Managerial & 1.0 & 3.3 & 3.5 & 7.0 & 6.3 & 1.6 \\
\hline Clerical/sales & 1.2 & 3.0 & 1.6 & 4.2 & 5.3 & 1.5 \\
\hline Labour & 41.3 & 17.4 & 28.8 & 9.3 & 19.0 & 54.5 \\
\hline Household worker & 0.4 & 2.4 & 0.4 & 2.2 & 2.7 & 1.0 \\
\hline Not working & 56.1 & 73.9 & 65.6 & 77.4 & 66.7 & 41.5 \\
\hline \multicolumn{7}{|l|}{ Standard of living ind $\mathrm{x}^{4}$} \\
\hline Low & 41.0 & 17.4 & 26.5 & 7.7 & 16.6 & 46.8 \\
\hline Medium & 47.1 & 50.4 & 48.8 & 39.9 & 48.9 & 45.2 \\
\hline High & 11.8 & 32.3 & 24.7 & 52.5 & 34.5 & 8.0 \\
\hline Total percent & 100.0 & 100.0 & 100.0 & 100.0 & $\mathbf{1 0 0 . 0}$ & 100.0 \\
\hline Number of women & $\mathbf{5 0 2 5 3}$ & 8730 & 4629 & 9947 & 3474 & 6687 \\
\hline
\end{tabular}

1 "Middle school complete" means 89 completed years of education, "high school complete" means 10 or more completed years of education.

2 Other religions include Buddhist, Jain, Jewish, Zorastrian, and others.

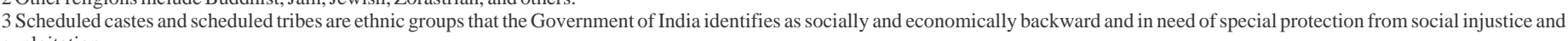
exploitation.

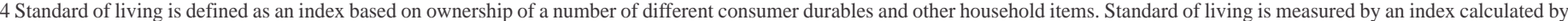

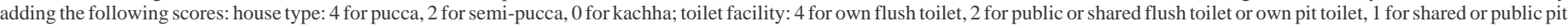

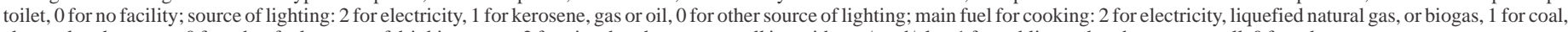

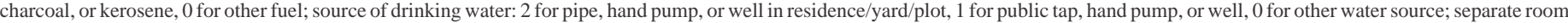

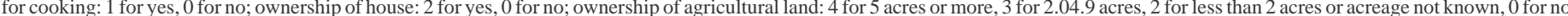

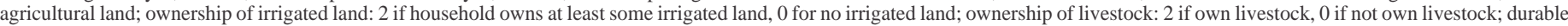

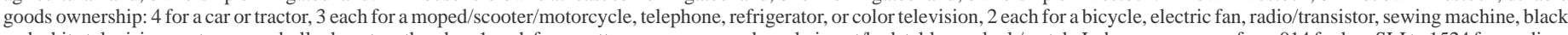

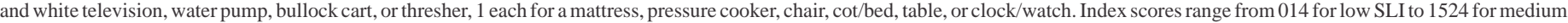
SLI to 2567 for high SLI.

Table 2: General health and morbidity condition among women aged 15- 49 years according to streams of migration and among non-migrant women, India, 1998-99

\begin{tabular}{|c|c|c|c|c|c|c|}
\hline \multirow[b]{2}{*}{ Health and Morbidity Condition } & \multicolumn{4}{|c|}{ Migrants according to stream of migration } & \multicolumn{2}{|c|}{ Non-migrant } \\
\hline & $\begin{array}{c}\text { Rural to } \\
\text { Rural }\end{array}$ & $\begin{array}{c}\text { Rural to } \\
\text { Urban }\end{array}$ & $\begin{array}{c}\text { Urban to } \\
\text { Rural }\end{array}$ & $\begin{array}{c}\text { Urban to } \\
\text { Urban }\end{array}$ & $\begin{array}{c}\text { Urban } \\
\text { non- } \\
\text { migrant }\end{array}$ & $\begin{array}{c}\begin{array}{c}\text { Rural } \\
\text { non- } \\
\text { migrant }\end{array} \\
\end{array}$ \\
\hline \multicolumn{7}{|l|}{ Nutritional status } \\
\hline Underweight $\left(\mathrm{BMI}<18.5 \mathrm{~kg} / \mathrm{m}^{2}\right)$ & 40.2 & 26.3 & 33.0 & 18.7 & 22.3 & 40.8 \\
\hline Overweight (BMI $\left.25-30 \mathrm{~kg} / \mathrm{m}^{2}\right)$ & 53.8 & 55.8 & 55.2 & 52.4 & 52.4 & 52.9 \\
\hline Obese $\left(\right.$ BMI $\left.>30 \mathrm{~kg} / \mathrm{m}^{2}\right)$ & 4.5 & 14.1 & 9.5 & 21.0 & 18.0 & 4.8 \\
\hline Normal & 1.5 & 3.8 & 2.4 & 7.9 & 7.3 & 1.5 \\
\hline \multicolumn{7}{|l|}{ Anaemia $^{2}$} \\
\hline Severe & 1.6 & 1.3 & 1.4 & 1.2 & 1.3 & 2.3 \\
\hline Moderate & 13.8 & 11.2 & 12.6 & 9.9 & 11.1 & 15.3 \\
\hline Mild & 34.1 & 30.1 & 32.4 & 29.5 & 30.1 & 32.6 \\
\hline No & 50.6 & 57.4 & 53.7 & 59.5 & 57.5 & 49.7 \\
\hline \multicolumn{7}{|l|}{ Morbidity condition $^{3}$} \\
\hline Asthma & 2412 & 2188 & 1988 & 1791 & 1699 & 3321 \\
\hline Tuberculosis & 723 & 527 & 692 & 392 & 403 & 778 \\
\hline Received treatment for & 85.8 & 93.3 & 100.0 & & & \\
\hline Tuberculosis $^{1}$ & & & & 91.4 & 92.3 & 80.8 \\
\hline Jaundice during the past 12 months & 4652 & 2864 & 4582 & 2394 & 1873 & 4835 \\
\hline Malaria during the past 3 months & 1262 & 1237 & 1318 & 986 & 865 & 1318 \\
\hline
\end{tabular}

1 Shown in percentage; Nutritional status shows the Body Mass Index (BMI) of women. Body Mass Index is defined as weight in kilograms divided by height in meters squared $(\mathrm{kg} / \mathrm{m} 2)$. Underweight: BMI $<18.5 \mathrm{~kg} / \mathrm{m} 2$; normal weight: $18.5-24.9 \mathrm{~kg} / \mathrm{m} 2$; overweight: $25.0-29.9 \mathrm{~kg} / \mathrm{m} 2$; obese: $>30.0 \mathrm{~kg} / \mathrm{m} 2$. Nutritional analysis excludes women pregnant at the time of the survey and those who gave birth in the 2 months preceding the survey.

2 Shown in percentage;

3Prevalence per 100000 population 
Table 3: Logistic regression result showing adjusted effects (odds ratios) of selected socio-economic, demographic and health status on different streams of migrant and non-migrant women aged 15- 49 years, India, 1998-99

\begin{tabular}{|c|c|c|c|c|c|c|}
\hline \multirow[b]{2}{*}{ Background characteristics } & \multicolumn{4}{|c|}{ Migrants according to streams of migration } & \multirow[b]{2}{*}{$\begin{array}{l}\text { Urban } \\
\text { non- } \\
\text { migrant }\end{array}$} & \multirow[b]{2}{*}{$\begin{array}{l}\text { Rur al non- } \\
\text { migrant }\end{array}$} \\
\hline & $\begin{array}{l}\text { Rural to } \\
\text { Rural }\end{array}$ & $\begin{array}{l}\text { Rural to } \\
\text { Urban }\end{array}$ & $\begin{array}{l}\text { Urban } \\
\text { to Rural }\end{array}$ & $\begin{array}{l}\text { Urban } \\
\text { to } \\
\text { Urban }\end{array}$ & & \\
\hline \multicolumn{7}{|l|}{$\begin{array}{c}\text { Age groups } \\
15-24^{\mathbf{R}}\end{array}$} \\
\hline $25-34$ & $0.834 * * *$ & $1.173^{* * *}$ & 0.962 & $1.322 * * *$ & 1.014 & $0.929 * *$ \\
\hline $35-49$ & $0.767 * * *$ & $1.168 * * *$ & $0.826^{* * *}$ & $1.550 * * *$ & 0.959 & $1.069^{*}$ \\
\hline \multicolumn{7}{|l|}{$\begin{array}{l}\text { Education } \\
\text { Illiterate }\end{array}$} \\
\hline Literate, $<$ middle school complete & $0.589 * * *$ & $1.186^{* * *}$ & $1.919 * * *$ & $1.894 * * *$ & $1.231 * * *$ & $1.216^{* * *}$ \\
\hline Middle school complete & $0.421 * * *$ & $1.164 * * *$ & $2.463 * * *$ & $2.896 * * *$ & $1.368 * * *$ & $1.213 * * *$ \\
\hline High school complete and above & $0.229 * * *$ & $0.906 * * *$ & $2.527 * * *$ & $5.252 * * *$ & $1.564 * * *$ & 1.107 \\
\hline \multicolumn{7}{|l|}{ Religion } \\
\hline \multicolumn{7}{|l|}{ Hindu ${ }^{\mathbf{R}}$} \\
\hline Muslims & $0.504 * * *$ & 1.016 & 0.940 & $1.701 * * *$ & $2.268 * * *$ & $2.251 * * *$ \\
\hline Christian & $0.371 * * *$ & $1.225^{* * *}$ & $1.230 * * *$ & $0.793 * * *$ & $1.754 * * *$ & $5.097 * * *$ \\
\hline Sikh & $2.987 * * *$ & $0.491 * * *$ & 1.022 & $0.560 * * *$ & $0.405 * * *$ & $0.086^{* * *}$ \\
\hline Others & $0.476 * * *$ & 1.006 & $1.518^{* * *}$ & $1.145^{*}$ & 0.890 & $3.572 * * *$ \\
\hline \multicolumn{7}{|l|}{ Scheduled caste ${ }^{\mathbf{R}}$} \\
\hline Scheduled tribe & $1.145 * * *$ & $0.568 * * *$ & $0.749 * * *$ & $0.580 * * *$ & 1.095 & $1.801 * * *$ \\
\hline Other backward class & $1.107 * * *$ & $0.847 * * *$ & 1.030 & $0.661 * * *$ & $1.213 * * *$ & $1.412 * * *$ \\
\hline Others & $1.163 * * *$ & $0.814 * * *$ & $0.909^{*}$ & 0.989 & $0.623 * * *$ & $1.106^{* *}$ \\
\hline \multicolumn{7}{|l|}{ Standard of living index } \\
\hline Medium & $0.740 * * *$ & $1.776^{* * *}$ & $1.078^{*}$ & $2.195 * * *$ & $0.822 * * *$ & $0.830 * * *$ \\
\hline High & $0.478 * * *$ & $2.373 * * *$ & 0.984 & $3.717 * * *$ & $0.628 * * *$ & $0.511 * * *$ \\
\hline \multicolumn{7}{|l|}{ Occupation } \\
\hline \multicolumn{7}{|l|}{ Professional/Technical/Managerial ${ }^{\mathbf{R}}$} \\
\hline Clerical/sales & $0.766 * * *$ & $1.489 * * *$ & $0.791^{*}$ & $1.260 * * *$ & 1.083 & 0.887 \\
\hline Labour & $2.015 * * *$ & $0.551 * * *$ & $0.842^{*}$ & $0.397 * * *$ & 1.062 & 1.064 \\
\hline Household worker & $0.238 * * *$ & $2.868 * * *$ & $0.407 * * *$ & $3.688 * * *$ & 1.108 & $1.743 * * *$ \\
\hline Not working & $1.573 * * *$ & $1.164 * *$ & $0.819 * *$ & $0.872 * * *$ & $0.692 * * *$ & $0.631 * * *$ \\
\hline \multicolumn{7}{|l|}{ Anaemia stat us } \\
\hline Not anaemic & & & & & & \\
\hline Anae mic & 1.018 & $0.906 * * *$ & 0.995 & $1.096 * * *$ & 0.991 & 0.967 \\
\hline \multicolumn{7}{|l|}{ Nutritional status } \\
\hline Underweight & $1.184 * * *$ & $0.877 * * *$ & 1.043 & $0.804 * * *$ & 0.937 & $0.921 * * *$ \\
\hline Overweight & $0.523 * * *$ & $1.130 * * *$ & $0.861 * * *$ & $1.708 * * *$ & $1.181 * * *$ & 0.976 \\
\hline Constant & 1.988 & 0.092 & 0.046 & 0.034 & 0.249 & 0.120 \\
\hline
\end{tabular}

Table 4: Maternal and reproductive health problems among women aged 15-49 years according to streams of migration and non-migrant, India, 1998-99

\begin{tabular}{|c|c|c|c|c|c|c|}
\hline \multirow[b]{2}{*}{ Health Problems } & \multicolumn{4}{|c|}{ Migrants according to streams of migration } & \multicolumn{2}{|c|}{ Non-migrant } \\
\hline & $\begin{array}{c}\text { Rural to } \\
\text { Rural }\end{array}$ & $\begin{array}{l}\text { Rural to } \\
\text { Urban }\end{array}$ & $\begin{array}{c}\text { Urban to } \\
\text { Rural }\end{array}$ & $\begin{array}{l}\text { Urban to } \\
\text { Urban }\end{array}$ & $\begin{array}{c}\text { Urban } \\
\text { non- } \\
\text { migrant }\end{array}$ & $\begin{array}{l}\text { Rural non- } \\
\text { migrant }\end{array}$ \\
\hline \multicolumn{7}{|l|}{ Maternal health problems } \\
\hline Ever had an abortion & 9.6 & 13.0 & 13.5 & 13.1 & 13.3 & 10.4 \\
\hline Ever had a terminated pregnancy & 18.7 & 22.0 & 21.8 & 24.3 & 22.4 & 18.6 \\
\hline Ever had a still birth & 3.5 & 2.1 & 2.5 & 1.4 & 2.4 & 2.3 \\
\hline \multicolumn{7}{|l|}{ Reproductive health problems } \\
\hline Itching & 18.1 & 17.6 & 16.4 & 15.6 & 13.4 & 15.6 \\
\hline Bad odour & 12.9 & 9.4 & 11.1 & 9.1 & 6.6 & 10.6 \\
\hline Abdominal pain & 19.7 & 18.7 & 18.5 & 15.7 & 14.8 & 19.9 \\
\hline Fever & 8.8 & 7.1 & 8.5 & 5.9 & 5.7 & 9.5 \\
\hline Other problems & 7.7 & 8.9 & 8.5 & 8.7 & 7.7 & 9.0 \\
\hline Pain or burning during urination & 18.7 & 17.1 & 18.6 & 14.9 & 10.1 & 12.6 \\
\hline Painful intercourse & 12.6 & 12.3 & 15.5 & 11.0 & 10.1 & 12.6 \\
\hline Blood visible after sex & 2.3 & 1.9 & 2.9 & 1.5 & 2.6 & 3.3 \\
\hline
\end{tabular}


Table 5: Knowledge related to STDs and AIDS among women aged 15-49 years according to streams of migration and nonmigrant, India, 1998-99

\begin{tabular}{|c|c|c|c|c|c|c|}
\hline \multirow[t]{2}{*}{ Knowledge related to AIDS/ STDs } & \multicolumn{4}{|c|}{ Migrants according to streams } & \multicolumn{2}{|c|}{ Non-migrant } \\
\hline & $\begin{array}{c}\text { Rural to } \\
\text { Rural }\end{array}$ & $\begin{array}{l}\text { Rural to } \\
\text { Urban }\end{array}$ & $\begin{array}{c}\text { Urban to } \\
\text { Rural }\end{array}$ & $\begin{array}{l}\text { Urban to } \\
\text { Urban }\end{array}$ & $\begin{array}{c}\text { Urban } \\
\text { non- } \\
\text { migrant }\end{array}$ & $\begin{array}{c}\text { Rural non- } \\
\text { migrant }\end{array}$ \\
\hline Ever heard of AIDS & 24.8 & 58.3 & 56.3 & 78.8 & 75.2 & 41.8 \\
\hline Received information from: & & & & & & \\
\hline Radio & 45.1 & 36.3 & 44.2 & 34.5 & 40.5 & 46.9 \\
\hline Television & 66.0 & 86.5 & 82.7 & 94.2 & 90.0 & 60.4 \\
\hline Cinema & 5.1 & 8.2 & 8.0 & 10.9 & 13.8 & 6.8 \\
\hline New spapers/magazines & 18.9 & 26.4 & 28.9 & 38.7 & 32.7 & 16.0 \\
\hline Posters/hoarding & 8.6 & 12.5 & 13.6 & 17.2 & 18.4 & 7.6 \\
\hline Clinic/health worker & 4.2 & 2.7 & 5.1 & 2.8 & 2.9 & 4.4 \\
\hline Schools/teachers & 1.0 & 0.8 & 1.0 & 1.1 & 0.8 & 1.3 \\
\hline Friends/relatives & 37.8 & 25.6 & 27.6 & 19.9 & 28.5 & 47.5 \\
\hline Adult education programs & 0.5 & 0.4 & 0.4 & 0.6 & 0.3 & 0.4 \\
\hline Other sources & 7.7 & 5.0 & 6.7 & 4.6 & 4.8 & 10.7 \\
\hline $\begin{array}{l}\text { Knowledge about avoidance of AIDS* } \\
\text { Percent who believes AIDS can be avoided } \\
\text { by }\end{array}$ & 62.7 & 66.6 & 69.0 & 74.2 & 75.5 & 64.2 \\
\hline Abstaining from sex & 6.1 & 6.2 & 7.4 & 9.8 & 4.8 & 2.9 \\
\hline Using condoms during sex & 13.5 & 19.7 & 20.3 & 31.8 & 21.3 & 9.0 \\
\hline Having only one sex partner & 37.7 & 37.6 & 40.5 & 41.7 & 50.6 & 40.9 \\
\hline Avoiding sex with commercial sex workers & 20.9 & 26.8 & 24.8 & 27.2 & 35.0 & 25.4 \\
\hline Avoiding sex with homosexuals & 2.4 & 3.6 & 2.3 & 3.8 & 4.8 & 2.1 \\
\hline Avoiding blood transfuse & 15.2 & 18.3 & 18.7 & 23.7 & 26.3 & 14.3 \\
\hline Avoiding injections/using clean needles & 24.1 & 29.5 & 30.9 & 36.9 & 35.1 & 24.3 \\
\hline Avoid I.V. drug use & 1.3 & 1.3 & 1.7 & 2.2 & 1.4 & 1.3 \\
\hline Sharing razors & 3.5 & 3.0 & 3.3 & 3.2 & 2.3 & 3.2 \\
\hline Other ways & 7.1 & 7.0 & 6.0 & 6.6 & 5.3 & 5.7 \\
\hline $\begin{array}{l}\text { Knowledge of condom for family planning } \\
\text { and STDs }\end{array}$ & 3.3 & 11.4 & 11.4 & 25.0 & 15.9 & 3.7 \\
\hline
\end{tabular}

* Includes only those women who had heard about AIDS

Table 6: Knowledge related to STDs and AIDS according to duration and stream of migrants and non-migrant women aged 15- 49 years, India, 1998-99

\begin{tabular}{|c|c|c|c|c|}
\hline \multirow[b]{2}{*}{$\begin{array}{l}\text { Knowledge related to AIDS or } \\
\text { STDs/ Duration of migration }\end{array}$} & \multicolumn{4}{|c|}{ Migrants according to streams of migration } \\
\hline & $\begin{array}{l}\text { Rural to } \\
\text { Rural }\end{array}$ & $\begin{array}{l}\text { Rural to } \\
\text { Urban }\end{array}$ & $\begin{array}{l}\text { Urban to } \\
\text { Rural }\end{array}$ & $\begin{array}{l}\text { Urban to } \\
\text { Urban }\end{array}$ \\
\hline \multicolumn{5}{|l|}{ Ever heard of AIDS } \\
\hline $1-2$ years & 32.5 & 58.8 & 71.2 & 82.8 \\
\hline $3-5$ years & 30.3 & 60.9 & 61.5 & 83.5 \\
\hline $6-9$ years & 26.7 & 64.9 & 58.7 & 83.1 \\
\hline 10-19 years & 23.6 & 58.6 & 51.4 & 79.2 \\
\hline 20 years and above & 20.4 & 52.5 & 43.6 & 68.3 \\
\hline Total & 24.8 & 58.3 & 56.3 & 78.8 \\
\hline \multicolumn{5}{|l|}{ Ways to avoid A IDS ${ }^{1}$} \\
\hline $1-2$ years & 67.2 & 70.7 & 68.7 & 77.5 \\
\hline $3-5$ years & 66.1 & 68.9 & 71.5 & 77.3 \\
\hline $6-9$ years & 66.5 & 70.2 & 70.8 & 76.6 \\
\hline 10-19 years & 62.2 & 66.8 & 67.1 & 73.4 \\
\hline 20 years and above & 56.1 & 59.9 & 67.5 & 67.5 \\
\hline Total & 62.7 & 66.6 & 69.0 & 74.2 \\
\hline \multicolumn{5}{|l|}{$\begin{array}{l}\text { Knowledge of condom for STDs } \\
\text { and family planning }\end{array}$} \\
\hline $1-2$ years & 5.9 & 13.1 & 18.0 & 29.0 \\
\hline 3-5 years & 5.6 & 14.1 & 15.2 & 32.0 \\
\hline $6-9$ years & 4.3 & 13.9 & 12.7 & 28.2 \\
\hline 10-19 years & 2.7 & 11.6 & 7.6 & 23.6 \\
\hline 20 years and above & 1.6 & 7.6 & 6.9 & 16.3 \\
\hline Total & 3.3 & 11.4 & 11.4 & 25.0 \\
\hline
\end{tabular}


proportion of other castes women were found among rural nonmigrants. This shows caste differential in migration in India.

Also the stream of migration differs according to the working status of women. Less proportion of working women was found to be migrating towards rural area (rural to rural and urban to rural) than towards urban area (urban to urban and rural to urban). $56 \%$ of not working women were migrating from rural to rural area compared to $74 \%$ from rural to urban area, and $77 \%$ from urban to urban area. In addition to this, women working in professional/technical/ managerial jobs or in clerical or sales jobs were migrating more towards urban area than towards rural area, whereas women working as labourer were migrating more towards rural area as higher proportion of labourers (58\%) were found among rural non-migrants.

A significant differential has been observed with standard of living of women and pattern of migration. Rural to rural, and rural to urban migration was dominated by women belonging to household with low and medium standard of living whereas urban to urban migration was dominated by women belonging to household with high standard of living. $41 \%$ percent of rural to rural migrants and $27 \%$ of urban to rural migrants were from low standard of living whereas only $8 \%$ and $17 \%$ of urban to urban, and rural to urban migrants respectively, belongs to a lower standard of living. However, 52\% of urban to urban migrants and $32 \%$ of rural to urban migrants were from high standard of living whereas only $12 \%$ and one-fourth from rural to rural, and urban to rural migrants respectively, were from higher standard of living respectively. On the other hand, $47 \%$ of the rural non-migrants were from households with a lower standard of living compared to only $17 \%$ of urban non-migrants. However, $35 \%$ of urban non-migrants were from high standard of living households compared to only $8 \%$ of rural non-migrants. This clearly shows the economic differential in the streams of migration. Poorer women were migrating towards rural areas whereas economically better off women were migrating towards urban areas.

\section{Health and morbidity condition of women according to streams of migration}

Table 2 presents general health situation and morbidity condition of migrant and non-migrant women according to streams of migration. Women migrating towards rural area were found more underweight than women migrating towards urban area, whereas women migrating towards urban area were more obese than women migrating towards rural area. Two-fifth of women migrating from rural to rural area, and one-third of women migrating from urban to rural area were found underweight, compared to two-fifth women migrating from urban to urban area and one-fourth rural to urban migrant women. However, obesity increased from $<2 \%$ among rural to rural migrant women to $8 \%$ among urban to urban migrant women. On the other hand, rural non-migrants shows similar pattern as rural to rural migrants and urban non-migrant also shows similar pattern as urban to urban migrants.

Regarding anemia status, it is found that, women migrating from rural to rural area were more mild anemic (34\%) than rural to urban or urban to urban area (30\%). Moderate and severe anemia also shows the similar pattern. Almost similar pattern like urban to urban migration was found in case of urban non-migrants and also similar pattern like rural to rural migration for rural non-migrants
Morbidity condition of women has been seen for asthma, tuberculosis, jaundice during the past 12 monthsandmalariaduring the past three months in per 100000 population. Prevalence of asthma was noticed more among rural to rural migrant women (2412) than rural to urban (2188), urban to urban (1791) and urban to rural migrant women (1988). However, among the urban non-migrant women, prevalence of asthma was lowest (1699) and it was highest among rural nonmigrants (3321).

Prevalence of tuberculosis was found more among women migrating towards rural area than women migrating towards urban area. The situation improves for women who were migrating from rural area to urban area than women who remains in rural area after migration, as the prevalence of tuberculosis was noticed 723 for rural to rural migrant women, whereas 527 for rural to urban migrant women. However, the condition deteriorates for the women who migrates from urban area to rural area than women migrating from urban to urban area, or even rural to urban area, as the prevalence of tuberculosis was noticed 692 for urban to rural migrant women compared to 392 for urban to urban migrant women and 527 for rural to urban migrant women. On the other hand, among the rural non-migrant women, prevalence of tuberculosis was found highest (778) whereas prevalence for urban non-migrant women was almost similar to urban to urban migrant women.

Prevalence of jaundice was found highest among rural to rural migrant women (4652) followed by urban to rural migrant women (4582), whereas it was least among urban to urban migrant women (2394). On the other hand, the prevalence of jaundice was lowest among the urban non-migrant women (1873) and highest among rural non-migrant women.

Prevalence of malaria follows the jaundice pattern. It was highest among urban to rural migrant women (1318) followed by rural to rural migrant women (1262), whereas it was found least among urban to urban migrant women (986). On the other hand, among the rural non-migrant women, prevalence of malaria was equal to urban to rural migrant women and it was least among urban non-migrant women (865).

Association between socio-economic demographic and health status of women and streams of migration

Table 3 presents the adjusted odds ratio for different streams of migration by selected socio-economic, demographic and health characteristics. The result of logistic regression substantiates that the stream of migration is influenced by the age, ethnicity, religion, standard of living, education, occupation, anemia status and nutritional status of women and the odds ratios varies significantly with different streams of migration. The likelihood of rural to rural migration decreases with age, education, standard of living and nutritional status of women. Also likelihood decreases among women engaged in household works with reference to women doing professional /technical/ managerial works, and among women of all the religious groups except Sikh women with reference to Hindu women and results are statistically significant. However, likelihood of rural to rural migration increases among scheduled tribes, other backward class and women belonging to other caste with reference to scheduled caste, and among women engaged as labourer with reference to women doing professional/technical/managerial jobs.

Almost reverse situation was found in case of rural to 
urban migration. The likelihood of rural to urban migration increases with age, standard of living, education and nutritional status of women. Also likelihood of rural to urban migration increases among household workers with reference to women working in professional/technical/managerial jobs and among Christian women with reference to Hindu women and results are statistically significant. However, likelihood of rural to urban migration significantly decreases among all the ethnic groups with reference to scheduled caste women, and also among women working as labourer with reference to women engaged in professional/technical/managerial jobs.

Migration from urban to rural areas shows relatively less significant predictors. The likelihood of urban to rural migration increases with education and among Christian and women belonging to other religion with reference to Hindu women. However, likelihood of urban to rural migration decreases with age and nutritional status of women and among all working women other than professional/technical/managerial jobs.

Almost similar pattern like rural to urban migration but with more gradient was found in case of urban to urban migration. The likelihood of urban to urban migration increases with age, education, standard of living and nutritional status of women. Women engaged as household worker and in clerical/sales works were more likely to migrate from urban to urban area with reference to women engaged in professional/technical/managerial jobs. Also, Muslim women and women belonging to other religion were more likely to migrate from urban to urban area with reference to Hindu women being highly statistically significant. However, likelihood of urban to urban migration decreases significantly among scheduled caste and other backward class women with reference to scheduled caste women, and also among women engaged as labourer and not working women with reference to women engaged in professional/technical/managerial jobs.

Urban non-migrants show somewhat reverse pattern than all types of migration streams. The likelihood of urban nonmigrant increases with education and higher nutritional status of women. However, likelihood of urban non-migrant decreases with standard of living. On the other hand, odds ratio for rural non-migrants were found not to be significant for women with higher education and higher nutritional status, which were significant in case of urban non-migrants also. Another very significant difference between urban non-migrants and rural nonmigrants is that, other caste women were found to be significantly less among urban non-migrant whereas significantly more among rural non-migrants with reference to scheduled castes women. Also, the likelihood of rural non-migrants increases among household worker with reference to women engaged in professional/technical/managerial jobs.

\section{Maternal and reproductive health of women according to different streams of migration}

Table 4 presents maternal and reproductive health problems among migrant and non-migrant women according to streams of migration. Maternal health problems has been seen in terms of the following: ever had an abortion, ever had a terminated pregnancy and ever had a still birth; and reproductive health problems includes: itching, bad odour, abdominal pain, fever and other problems, pain or burning sensation during urination, painful intercourse and blood visible after sex. A mixed pattern of maternal health problems and reproductive health problems has been observed according to different streams of migration.

Women migrating towards urban area or migrating from urban area has ever experienced higher abortion (for every stream more than 13\%) than rural to rural area (10\%). Similarly, ever experience of pregnancy termination was experienced more among women who were migrating towards urban area or migrating from urban area (for every stream $\geq 22 \%$ ) compared to women migrating from rural to rural area (19\%). However, migrants from rural to rural area had experienced higher rate of stillbirths than other streams. On the other hand, rural nonmigrant women have experienced less abortion or pregnancy termination than urban non-migrant women.

Most of the reproductive health problems were found to be higher among rural to rural migrant women than rural to urban migrant women. Itching, bad odour, abdominal pain, fever and pain or burning sensation during urination was experienced by 18 , $13,20,9$, and $19 \%$ of rural to rural migrant women, respectively compared to $18,9,19,7$, and $17 \%$ of rural to urban migrant women, respectively. Urban to rural migrant women were also having more reproductive health problems than urban to urban migrant women. On the other hand, among urban non-migrant women, comparatively less reproductive health problems were found than rural non-migrant women.

Knowledge and awareness of AIDS among women Table 5 presents knowledge related to STDs and AIDS among migrant women according to different streams of migration and also among the non-migrant women. Very less percentage of women who migrated from rural to rural area had heard about AIDS compared to women belonging to other streams of migration. One in four women migrating from rural to rural area have ever heard of AIDS compared to four in five urban to urban migrant women, 58\% rural to urban migrant women and 56\% urban to rural migrant women. On the other hand, $42 \%$ of rural non-migrant women had ever heard of AIDS as compared to three-fourth of urban non-migrant women. Among women who had heard about AIDS, majority had heard the message from television followed by radio and newspaper.

However, friends and relatives were better source of information about AIDS than newspaper for the rural to rural migrant women. Among rural and urban non-migrant women, the sources of information about AIDS were found similar.

Among those women who had heard about AIDS, again less percentage of women migrating from rural to rural area $(63 \%)$ knew ways to avoid AIDS, compared to $67 \%$ women migrating from rural to urban area, 69\% women migrating from urban to rural area which further increases to $74 \%$ among urban to urban migrant women. Again, having only one sex partner was found to be the predominant way to avoid AIDS followed by avoiding injections and avoiding sex with commercial sex workers for all the streams of migration and also among the non-migrant women.

Knowledge of condom use for family planning and avoidance of sexually transmitted infections (STDs), significantly varies among women according to their streams of migration. About one in four urban to urban migrant women were having knowledge of condom for family planning and STDs compared to only $3 \%$ of rural to rural migrant women and one in ten in both (rural to urban and urban to rural migrant women). On the other hand, $16 \%$ of the urban non-migrant women were having knowledge of condom for family planning and STDs compared to only $4 \%$ among rural non-migrant women. 


\section{Knowledge and awareness of AIDS among women by duration of migration}

Table 6 presents knowledge related to STDs and AIDS by duration and streams of migration and among non-migrant women. In case of rural to rural migrant women, knowledge has been significantly decreased from $33 \%$ among women who stayed for 1-2 years to $27 \%$ among women who stayed for 6 to 9 years and further to $20 \%$ among women who stayed for more than 20 years. This shows that women who migrated recently had more knowledge about AIDS. However, in case of rural to urban migrant women, knowledge has been significantly increased with exposure to stay in urban area. Knowledge of AIDS increased from 59\% among women who stayed for 1-2 years, to $61 \%$ among women who stayed for 3 to 5 years and further to $65 \%$ among women who stayed for 6 to 9 years. On the other hand, in case of urban to rural migrant women, knowledge has been significantly decreased with exposure to stay in rural area. Knowledge of AIDS decreased from $71 \%$ among women who stayed for 1-2 years, to $62 \%$ among women who stayed for 3 to 5 years and further decreased to $59 \%$ among women stayed for 6 to 9 years. But in case of urban to urban migrant women, knowledge of AIDS was more or less constant in different duration of stay. It is a noticeable point that, in all the streams of migration, knowledge of AIDS with duration of stay more than 10 years and above is comparatively lower than women who are recent migrants. It may be because of the fact that the epidemic of AIDS in India had been seen from the last 10 years and women of older cohort may have less knowledge about HIV/AIDS.

Among those migrant women who had heard about AIDS, knowledge about the ways to avoid AIDS also differs according to different streams of migration. It shows almost a similar pattern like ever heard of AIDS. However, duration of migration of women except 10 years and above in all the streams of migration does not show significant differential in knowledge about the ways to avoid AIDS.

Knowledge of condom for family planning and STDs, significantly varies among migrant women according to the streams of migration and also by the duration of migration. Urban to urban migrant women has the highest knowledge of condom for family planning and STDs followed by urban to rural migrant women, and rural to urban migrant women. Recent urban to rural migrant women have more knowledge of condom for family planning and STDs than older duration migrant women, as $18 \%$ among 1-2 years migrants have knowledge of condom for family planning and STDs compared to $15 \%$ among 3-5 years migrants and further to only $13 \%$ among 6-9 years migrants. However, in other streams of migration, knowledge of condom for family planning and STDs has not significantly varied by duration of migration, except for 10 years and above. With duration of stay more than 10 years and above, in all the streams of migration, knowledge of condom for family planning and STDs were comparatively lower than recent duration migrants.

\section{DISCUSSION}

It is evident from this study that in India, still rural to rural migration of women is predominant. Streams and duration of migration plays a key role in morbidity and health status of women. The pattern of different diseases and health status of women according to the streams of migration and also among the non-migrants shows that, in most of the cases, the situation of rural non-migrants is worst but situation of women migrating towards urban area shows improvement in their health condition.
Regarding health outcome prevalence, we found women migrating towards rural area were more underweight than women migrating towards urban area whereas it is reverse for obesity. However, women migrating from rural to rural area were more anaemic than women migrating from rural to urban area. Significant differences were also found for morbidity conditions like asthma, tuberculosis, jaundice, malaria, and some reproductive health problems among women according to streams of migration. Women migrating from rural to rural area had experienced more health problems compared to women migrating from rural to urban area. It is evident from the study that, rural non-migrant women have poor health status than urban non-migrant women. Also women who migrate from rural to urban area, their health status improves whereas women who migrate from urban to rural area their health status deteriorates. It might be because of the presence of better health care facilities in urban areas. Also it is evident from the study that, if a woman migrates from urban to rural area, their health status significantly deteriorates which further indicates the inadequacy of the health care facilities in rural India. This indirectly indicates the lacunae in the health care facilities in rural India.

In case of most of the maternal and reproductive health problems, women migrating towards rural areas are having relatively more of the reproductive and maternal health problems than women migrating towards urban areas. However, when women migrate towards urban areas, situation gets little better. Similarly, knowledge of AIDS also significantly differs according to the stream and duration of migration. Ever heard of AIDS or knowledge to avoid AIDS was significantly less among rural nonmigrant women than urban non-migrant women. Further, migration towards urban area enhanced the knowledge about AIDS whereas if migrating towards rural area knowledge to avoid AIDS decreases. This indicates poor information-educationcommunication programme in rural India. Women migrating towards urban area have better knowledge related to AIDS whereas the knowledge of AIDS decreases among women migrating towards rural areas. It shows ineffective IEC activities in rural India. The results are important from the policy point of view. Urban health infrastructure should be translated in the rural areas of India and also the IEC programmes related to AIDS should be made more strengthened and effective through television, radio and also through the school teachers to reach the rural masses in India

\section{ACKNOWLEDGMENT}

Authors are thankful to Prof. Kamla Gupta for useful comments on an earlier draft of the paper. SA is supported by a Wellcome Trust Strategic Award Grant No Z/041825.

\section{REFERENCES}

1. Hugo GE. Population Distribution and Migration, Proceedings of the United Nations Expert Meeting on Population Distribution, Santa Cruz, Bolivia. 18-22 January, 1993.

2. Mehta S. Spatial mobility in India: Evolving patterns, emerging issues and implications. Population Geography 1990;12(1\& 2).

3. Smita RC, Chandna S.Migration patterns in India. Population Geography 1991; 139(1-2):31-52.

4. Gill MS. Patterns of migration in an under-developed tract: Hissar district of Haryana, 1951-1971. Geographical Review 
of India 1981;43(2):105-121.

5. Lusome R, Bhagat RB. Trends and Patterns of Internal Migration in India, 1971-2001. Paper presented at the Annual Conference of Indian Association for the Study of Population (IASP) during 7-9 June, 2006, Thiruvananthapuram, India.

6. Singh JP. Migration in India: A review. Asian and Pacific Migration Journal 1992; 1(1):168-192.

7. Libee MJ, Sopher DE. Marriage migration in rural India, In: People on the move, Kosinski and Prothero (eds), London Methuen and Co. Ltd. Page 347-359, 1975.

8. Weiner M. Sons of the Soil: Migration and Ethnic Conflict in India. Princeton University Press, Princeton, 1978.

9. Ravenstein EG. The Laws of Migration. Journal of the Statistical Society $1885 ; 40(2): 167-199$.

10. Brockerhoff M. Child survival in big cities: The disadvantages of migrants. Social Science and Medicine 1995;40:1371.

11. Brockerhoff $M$. The impact of rural-urban migration on child survival. Health Transition Review 1994; 4:127.
12. Brockerhoff $M$. Rural to urban migration and child survival in Senegal. Demography 1990; 27:601.

13. Brockerhoff M, Eu H. Socio-economic and demographic determinants of female rural - urban migration in SubSaharan Africa. International Migration Review 1993;27:557.

14. Singh GB. Educational differentials in city ward migration in India. Canadian Studies in Population 1987;15(1):87-99.

15. Greenward MJ. The influence of family and friends on geographic labour mobility in a Less Developed country: The case of India. Review of Regional Studies 1971: 3(1):27-36.

16. Epstein TS. South India: Yesterday, Today and Tomorrow", London, Macmillan, 1973.

17. Iglesias E. et al. Women, International migration and selfreported health: A population-based study of women of reproductive age. Social Science and Medicine 2003;56(1):111-124.

18. International Institute for Population Sciences (IIPS), Macro ORC. National Family Health Survey (NFHS-2), 1998-99: India. Mumbai: International Institute for Population Sciences, 2000. 